\title{
ULTRASSONOGRAFIA E RESSONÂNCIA MAGNÉTICA: ESTUDO COMPARATIVO NO DIAGNÓSTICO DA ESTEATOSE EM OBESOS GRAU III
}

\author{
Gabriela Villaça Chaves*1, Sílvia Elaine Pereira', Carlos José Saboya', Caroline Cortes', Rejane Ramalho' \\ Trabalho realizado na Clínica Carlos Saboya, Rio de Janeiro, RJ
}

\author{
*Correspondência: \\ Av. Pau-Brasil, 211 -Bl. J - \\ Ilha da Cidade Universitária \\ Rio de Janeiro - RJ \\ CEP 21949-900 \\ Fax: (0xx21) 2280-8343/ \\ (21) 93170281 \\ gabrielavc@gmail.com
}

\begin{abstract}
RESUMO
OBJEtIvos. Avaliar a concordância entre a ressonância magnética (RM) e a ultrassonografia abdominal (USG) no diagnóstico da doença hepática gordurosa não-alcoólica (DHGNA), bem como a concordância entre cada método com o exame padrão-ouro histopatológico.

Métodos. A população estudada foi constituída por 145 pacientes com obesidade grau III (IMC $\geq 40 \mathrm{Kg} / \mathrm{m}^{2}$ ), de ambos os sexos. O diagnóstico da DHGNA foi realizado por ultrassonografia e ressonância magnética. Em uma subamostra foi realizado o diagnóstico por biópsia hepática $(n=40)$. Para avaliar a concordância entre os diagnósticos por USG e RM, utilizou-se o coeficiente Kappa.

Resultados. Observou-se uma concordância fraca entre os dois métodos (Kappa ajustado=0,27; IC 95\%=0,07-0,39). Foi encontrada uma concordância moderada entre o diagnóstico da doença por USG e biópsia hepática, com 83,3\% de resultados concordantes e Kappa ajustado de 0,67. Já a concordância entre o diagnóstico por RM e histopatológico foi ausente, com 53,6\% de resultados concordantes e Kappa ajustado de 0,07.

ConcLusão. A boa concordância encontrada entre o diagnóstico realizado pela USG e a biópsia hepática reforça a necessidade de condução de mais estudos como os que vêm recomendando uma padronização da avaliação diagnóstica por USG como forma de minimizar a necessidade da realização da biópsia hepática para diagnóstico de formas mais graves da doença.
\end{abstract}

UniteRmos: Ultrassonografia abdominal. Ressonância magnética. Obesidade grau III. Esteatose hepática.

\section{INTRODUÇÃO}

A DHGNA apresenta um amplo aspecto histológico, que resulta da deposição de triglicerídeos nos hepatócitos. Compreende um espectro de alterações patológicas semelhantes às observadas nas hepatopatias alcoólicas, mas ocorrendo em indivíduos não-etilistas. Essas alterações variam de simples esteatose a esteato-hepatite não-alcoólica (EHNA), fibrose e cirrose!

Os métodos de imagem como a ultrassonografia (USG), ressonância magnética (RM) e tomografia computadorizada (TC) são úteis na detecção de esteatose moderada a grave. Entretanto, a acurácia da avaliação radiológica da DHGNA e sua utilidade no manejo da doença carecem de maiores informações e a habilidade destas modalidades radiológicas em distinguir as diferentes formas de DHGNA não é conhecida ${ }^{2}$.

A sensibilidade e especificidade da ultrassonografia abdominal em detectar mais de 33\% de esteatose está entre 60\% e 94\% e 88 e $95 \%$, respectivamente. Entretanto, com o aumento do IMC esta sensibilidade e especificidade caem para 49\% e 75\%, respectivamente, em indivíduos com obesidade grau $1 \mathrm{I}^{3}$.
A RM é mais sensível que a USG e a TC na avaliação da esteatose hepática. Entretanto, devido ao modesto aumento na acurácia do diagnóstico e, em contrapartida, ao grande aumento no custo da realização deste exame, a USG é o método mais usado de diagnóstico por imagem ${ }^{4}$. Porém, em indivíduos com obesidade grau III, a grande quantidade de gordura na região abdominal pode dificultar a avaliação da esteatose na USG, podendo ser mais indicada a utilização da RM.

Nenhuma modalidade de diagnóstico por imagem é capaz de detectar fibrose e inflamação hepática, não podendo ser feita através destes métodos a diferenciação entre os subtipos histológicos da esteatose hepática não-alcoólica relativamente benigna e as formas mais agressivas da $E H N A^{5}$. Para essa finalidade é necessária a realização da biópsia hepática ${ }^{6}$.

Saadeh et al ${ }^{2}$, num estudo que comparou a utilidade dos métodos de avaliação radiológica na avaliação da esteatose hepática, observaram que nenhum dos métodos diagnósticos foi capaz de detectar pacientes com diagnóstico histopatológico de EHNA. Além disso, só foi possível detectar a presença de esteatose nos casos em que havia mais de 33\% de gordura 
depositada no figado. A USG e a TC tiveram sensibilidade de 100\% e 93\% para detectar mais de 33\% de acúmulo de gordura hepática. Nenhum dos métodos diagnósticos foi capaz de detectar presença de corpúsculos de Mallory, degeneração balonizante dos hepatócitos e fibrose, importantes no estabelecimento do diagnóstico da EHNA.

Dessa forma, os achados histológicos permanecem o padrão-ouro para estabelecimento do diagnóstico da doença, já que a acurácia dos componentes clínicos, importantes para o diagnóstico da doença, também ainda não estão bem estabelecidos?.

Há, no entanto, grande discussão no sentido de esclarecer qual população de pacientes se beneficiaria com a realização da biópsia hepática, devido ao custo e riscos deste procedimento, bem como a ausência de uma terapia espećíica de tratamento para a doençą ${ }^{8}$.

Dessa forma, o objetivo do presente estudo foi avaliar a concordância entre a RM e a USG no diagnóstico da DHGNA, bem como a acurácia destes métodos através da utilização do exame histopatológico como padrão-ouro.

\section{Métodos}

Para avaliação do estado nutricional foram utilizados os indicadores peso e altura. O peso corporal foi mensurado por meio de balança eletrônica tipo plataforma (welmy), com capacidade máxima de $300 \mathrm{Kg}$. A estatura foi obtida mediante a utilização de estadiômetro, com o indivíduo em pé, descalço, com os calcanhares juntos, costas eretas e os braços estendidos ao lado do corpo ${ }^{9}$. O IMC foi calculado a partir da seguinte fórmula: peso corporal atual $(\mathrm{kg}) /$ estatura $\left(\mathrm{m}^{2}\right)^{9}$. Os pontos de corte adotados foram os recomendados pela $\mathrm{WHO}^{10}$ para classificação de eutrofia e sobrepeso, sendo os integrantes do estudo classificados em obesidade grau III, com IMC $\geq 40,0 \mathrm{~kg} / \mathrm{m}^{2}$. O presente estudo propôs a categorização dos indivíduos em sub-classes de IMC, sendo a amostra distribuída em classes intervalares de $5 \mathrm{~kg} / \mathrm{m}^{2}$, dando origem a cinco faixas: faixa I para aqueles entre 40 e $44,9 \mathrm{~kg} / \mathrm{m}^{2}$, faixa 2 aqueles entre 45 e 49,9 $\mathrm{kg} / \mathrm{m}^{2}$, faixa 3 entre 50 e $54,9 \mathrm{~kg} / \mathrm{m}^{2}$, faixa 4 aqueles entre 55 e 59,9 $\mathrm{kg} / \mathrm{m}^{2}$, e faixa 5 para aqueles entre 60 a $64,9 \mathrm{~kg} / \mathrm{m}^{2}$.

Foram utilizados os exames de diagnóstico por imagem para diagnóstico da DHGNA: USG e RM, avaliados por um mesmo membro da equipe de pesquisa". Em uma subamostra da população estudada, foi realizada a avaliação histológica por biópsia hepática'12.

A avaliação histológica ocorreu na subamostra composta por 40 pacientes submetidos ao processo cirúrgico para o tratamento da obesidade, por meio da retirada de $4 \mathrm{~mm}$ de espessura do lobo esquerdo do fígado, obtida através de punção com agulha (Biópsia percutânea), tendo sido as biópsias realizadas pelo cirurgião. A avaliação foi realizada por um mesmo patologista, que não tinha conhecimento dos dados clínicos e bioquímicos dos pacientes, através da coloração das peças por hematoxilina-eosina, tricrômico de Masson e Perl's. O hematoxilinaeosina permite visualizar de forma geral a arquitetura acinar, infiltrados inflamatórios e alterações em hepatócitos. O Masson verifica a presenca de fibrose, seja portal, peri-sinusoidal ou ao redor de veias centrolobulares. Já o Perl's verifica a presença de depósitos de ferro ${ }^{12}$.

A graduação da DHGNA e estadiamento da fibrose hepática foi dada segundo a proposta de Brunt et al.,'2. A graduação foi realizada considerando a presença de esteatose macrovesicular (esteatose simples) e a atividade necroinflamatória (presença de EHNA), sendo:

Esteatose macrovesicular - Grau 0: sem estatose; Grau I (leve): $<33 \%$ de acúmulo de gordura nos hepatócitos; Grau 2 (moderada): entre 33\% e 66\% dos hepatócitos afetados; Grau 3 (grave): > 66\% dos hepatócitos afetados.

Atividade Necroinflamatória (EHNA) - Grau I (leve): esteatose predominantemente macrovesicular, envolvendo mais de $66 \%$ dos lóbulos, presença ocasional de balonização dos hepatócitos na Zona 3 , inflamação lobular dispersa com inflamação aguda leve (células polimorfonucleares) e ocasionalmente inflamação crônica (células mononucleares), ausência ou presença leve de inflamação portal; Grau 2 (moderada): esteatose em qualquer grau, balonização evidente na Zona 3 dos hepatócitos, presença de células polimorfonucleares associadas aos hepatócitos balonizados, fibrose pericelular, podendo ocorrer inflamação crônica leve, inflamação portal e intra-acinar leve a moderada; Grau 3 (grave): esteatose paracinar envolvendo mais de $66 \%$ dos lóbulos, com presença de esteatose macro e microvesicular, balonização predominantemente na Zona 3 dos hepatócitos, inflamação lobular aguda e crônica dispersas, células polimorfonucleares podem estar concentradas na Zona 3 de balonização, presença de fibrose persinusoidal e inflamação portal leve a moderada.

A análise estatística foi realizada pelo programa estatístico SPSS, versão 10.0 .

A concordância entre os métodos radiológicos de diagnóstico da DHGNA e entre cada método com o padrão-ouro biópsia hepática foi medida pelo cálculo do coeficiente de Kappa tradicional (IC 95\%). O coeficiente de Kappa ajustado pela prevalência (IC 95\%) foi calculado a partir do programa PEPI, disponível na Internet (http://www.usdinc.com/pepi.html). A concordância medida pelo Kappa seguiu a orientação da literatura especializada: kappa < 0, 10 ausente; de 0, I I a 0,40 fraca; de 0,4I a 0,60 discreta; de 0,6I a 0,80 moderada; de 0,8I a 0,99 substancial e 1,00 concordância perfeita (Fleiss, 1981; Streiner \& Norman, 1995).

Este trabalho teve aprovação do Comitê de Ética e Pesquisa do Hospital Universitário Clementino Fraga Filho.

\section{Resultados}

A amostra foi composta de I 45 pacientes, sendo 44 (30,3\%) do sexo masculino e I0I (69,7\%) do sexo feminino. A média de idade dos indivíduos foi de 36,5 $\pm 11,7$ anos, variando de 19 anos a 64 anos.

A prevalência de DHGNA no grupo estudado foi de $71 \%$, considerando o diagnóstico por quaisquer dos dois métodos de imagem utilizados (RM ou USG). O diagnóstico da DHGNA foi positivo em 75\% e 69,3\% dos homens e mulheres, respectivamente, sem diferença estatística $(p=0,553)$ (Tabela I).

Pode-se observar um aumento na prevalência da DHGNA nas faixas etárias mais elevadas ( $p=0,034$, teste Qui quadrado). É possível observar ainda uma maior prevalência da DHGNA conforme aumenta o IMC, ainda que sem significância estatística $(p=0,388$, teste Qui quadrado).

Nas classes mais elevadas de IMC, especialmente entre as faixas de $50-54,9 \mathrm{Kg} / \mathrm{m}^{2}$ e $55-59,9 \mathrm{Kg} / \mathrm{m}^{2}$, a USG diagnosticou um maior 


\begin{tabular}{|c|c|c|c|c|}
\hline \multicolumn{5}{|c|}{$\begin{array}{l}\text { Tabela I - Freqüência da DHGNA segundo sexo, faixa etária e subclasse de } \\
\text { IMC, de acordo com o método de diagnóstico por imagem utilizado }\end{array}$} \\
\hline Categorias & $n$ & USG ou RM & USG & RM \\
\hline \multicolumn{5}{|l|}{ Sexo } \\
\hline Feminino & 101 & $69,5 \%$ & $53,4 \%$ & $52,1 \%$ \\
\hline Masculino & 44 & $75,0 \%$ & $69,7 \%$ & $63,1 \%$ \\
\hline \multicolumn{5}{|c|}{ Idade (anos)* } \\
\hline $19-30$ & 51 & $62,7 \%$ & $49,0 \%$ & $47,7 \%$ \\
\hline $31-50$ & 65 & $76,9 \%$ & $67,2 \%$ & $61,6 \%$ \\
\hline $51-70$ & 29 & $85,0 \%$ & $70,0 \%$ & $61,1 \%$ \\
\hline \multicolumn{5}{|c|}{ Subclasse de IMC $\left(\mathrm{Kg} / \mathrm{m}^{2}\right)$} \\
\hline $40-44,9$ & 85 & $69,4 \%$ & $53,6 \%$ & $54,5 \%$ \\
\hline $45-49,9$ & 34 & $67,6 \%$ & $55,9 \%$ & $54,8 \%$ \\
\hline $50-54,9$ & 16 & $81,2 \%$ & $75,0 \%$ & $50,0 \%$ \\
\hline $55-59,9$ & 7 & $85,7 \%$ & $85,7 \%$ & $66,6 \%$ \\
\hline $60-64,9$ & 3 & $66,6 \%$ & $66,6 \%$ & $66,6 \%$ \\
\hline
\end{tabular}

*Classificação da idade segundo IOM (200 I).

IMC = Índice de Massa Corporal; USG = Ultrassonografia; RM= Ressonância magnética USG ou RM= Diagnóstico positivo considerando-se quaisquer dos dois métodos utilizados

percentual de DHGNA ( $p=0,050$, teste Qui quadrado), quando comparado com a RM.

Na subamostra que foi realizada biópsia hepática para avaliação histológica da DHGNA $(n=40), 100 \%$ tinham o diagnóstico de DHGNA, sendo 16 pacientes (40\%) com esteatose leve, sete pacientes (17,5\%) com esteatose moderada, I5 pacientes (37,5\%) com EHNA e dois pacientes $(5,0 \%)$ com cirrose hepática.

Para avaliar a concordância entre os diagnósticos por USG e RM, utilizou-se o coeficiente Kappa ajustado pela prevalência da doença. Observou-se uma concordância fraca entre os dois métodos (Kappa ajustado=0,27; IC 95\%=0,07-0,39). A concordância encontrada foi de $63,4 \%$.

Não foi possível realizar testes de sensibilidade e especificidade, assim como os valores preditivos positivos e negativos para cada exame radiológico, uma vez que não houve resultados negativos para a doença no exame histopatológico, utilizado como o padrão-ouro para diagnóstico da DHGNA. Dessa forma, não há como calcular os possíveis falsos-positivos ou falsos-negativos.

Contudo, foi calculado o grau de concordância entre a USG e RM com o resultado obtido no exame histopatológico, através do coeficiente Kappa. A totalidade dos indivíduos que realizaram biópsia $(n=40)$ tinha DHGNA em algum grau. Foi encontrada uma concordância moderada entre o diagnóstico da doença por USG e biópsia hepática, com 83,3\% de resultados concordantes e Kappa ajustado de 0,67. Já a concordância entre o diagnóstico por RM e histopatológico foi ausente, com 53,6\% de resultados concordantes e Kappa ajustado de 0,07.

Foi possível identificar ainda que, na maioria dos casos em que a USG ou a RM não identificaram a doença, o resultado da biópsia hepática foi o de esteatose leve (60\% e 69,2\%, respectivamente).

\section{Discussão}

Os resultados da biópsia hepática $(n=40)$ demonstraram que 100\% dos indivíduos tinham DHGNA, com 40\% apresentando esteatose simples, 17,5\% esteatose moderada, 37,5\% EHNA e 5\% com cirrose hepática. A prevalência da EHNA na populaçãa em geral gira em torno de 3\%, aumentando para 20\%-40\% em indivíduos obesos ${ }^{13}$. Chitturi et al. ${ }^{14}$, estudando 30 indivíduos com obesidade grau III, encontraram prevalência de DHGNA confirmada por histologia em 96\% da amostra, com 56\% apresentando EHNA. No Brasil, Almeida ${ }^{15}$ avaliou 60 indivíduos com obesidade grau III por critério histológico e encontrou prevalências de DHGNA semelhantes às do presente estudo: $46,7 \%, 36,7 \%$ e $5 \%$ de esteatose simples, EHNA e cirrose, respectivamente.

A verdadeira prevalência da DHGNA na obesidade grau III ainda é pouco clara e pode estar sendo subestimada, principalmente pela falta de um teste diagnóstico mais preciso. As anormalidades clínicas e bioquímicas características da DHGNA não permitem um diagnóstico acurado das formas mais graves da doença, permanecendo a biópsia hepática como o padrão-ouro para este fimm ${ }^{16,17}$. Na prática clínica, há a necessidade da diferenciação entre a EHNA e a esteatose simples, pelo fato de a EHNA ser uma forma mais agressiva da DHGNA e poder progredir para fibrose hepática e estágios irreversíveis da doença hepática ${ }^{18}$.

No presente estudo, a DHGNA foi diagnosticada através de métodos de imagem, que possuem menor sensibilidade, uma vez que é necessário um mínimo de 33\% de acúmulo de gordura nos hepatócitos para que esta seja detectável por estes exames². Além disso, as modalidades radiológicas não são capazes de distinguir as diversas formas de DHGNA, o que é fundamental no prognóstico da doença, visto que cerca de $15 \%$ a $20 \%$ dos indivíduos com esteatose simples evoluem para EHNA ${ }^{19}$.

Confrontando o diagnóstico da DHGNA entre os testes radiológicos utilizados no presente estudo com o exame histopatológico, observou-se que a USG detectou mais casos da doença que a RM. A concordância entre a USG e o padrão-ouro foi forte, enquanto a concordância deste com a RM foi fraca. A concordância entre os dois métodos radiológicos foi fraca, confirmando o maior poder diagnóstico da USG. Contudo, quando se considerou o estadiamento da DHGNA, observou-se que, nos dois testes de imagem, o número de erros no diagnóstico foi mais elevado na esteatose leve, corroborando os achados de Saadeh et al. ${ }^{2}$, que encontraram um ponto de corte de 33\% de acúmulo de gordura no tecido hepático para que a doença fosse detectável pelos testes radiológicos em obesos grau III.

A USG é um exame vantajoso com relação à RM no diagnóstico da doença, por ser de mais baixo custo, de mais fácil acesso ao paciente, além de não apresentar riscos. $\mathrm{A} \mathrm{OMS}^{20}$ recomenda a utilização de métodos diagnósticos com essas características, por serem de mais fácil utilização em estudos populacionais. Mottin et al. ${ }^{21}$ mostraram sensibilidade e especificidade da USG de 49, $1 \%$ e $75 \%$, respectivamente, com valor preditivo positivo de $95,4 \%$, indicando a validade deste teste diagnóstico na obesidade grau III. A baixa sensibilidade do teste foi atribuída à dificuldade da realização do exame em indivíduos com obesidade grau III, pela grande quantidade de gordura na região 
abdominal. Entretanto, a USG pode detectar a presença da esteatose e demonstrar de forma confiável o grau de redução desta após restrição energética ${ }^{21,22,23}$.

Poucos estudos comparam a diferenciação entre EHNA e esteatose simples por exame de USG. Saadeh et al. ${ }^{2}$ sugerem que não existe diferença aparente entre as diferentes formas da DHGNA ao exame radiológico. Entretanto, no referido estudo, a avaliação foi limitada a um pequeno número de casos e somente achados qualitativos da gordura hepática foram descritos.

Liang et al. ${ }^{24}$ propuseram um sistema quantitativo que utiliza vários parâmetros da USG e sugeriram um score que determinasse a eficácia deste exame na diferenciação da EHNA. O sistema foi baseado nos diversos graus de textura acústica e ecogenicidade do parênquima, veias portas, veias hepáticas e vias biliares intra-hepáticas e extra-hepáticas para definir a gravidade do acúmulo de gordura hepático, comparando os resultados com o exame histopatológico. O sistema proposto se correlacionou significativamente com os diagnósticos de esteatose simples, fibrose e EHNA pelo exame histológico. Foi determinado ainda um ponto de corte para predizer a presença de EHNA com valores de sensibilidade, especificidade, valor preditivo positivo e acurácia de $72 \%$, 86\%, 93\% e 76\%, respectivamente. Os autores concluíram que este sistema mostrou boa sensibilidade e especificidade na detecção da presença de EHNA em obesos grau III, podendo ser útil na seleção dos pacientes que merecem mais atenção no acompanhamento clínico sem a necessidade da realização de biópsia hepática.

Bernal-Reyes et al. ${ }^{25}$ sugerem que a combinação da USG com níveis séricos elevados de alanina aminotransferase (ALT) possui uma utilidade maior no diagnóstico da NASH em indivíduos que possuem maior risco ao desenvolvimento desta forma de DHGNA, como diabéticos e obesos (Sensibilidade $=80 \%$, especificidade $=100 \%$, valor preditivo positivo $=100 \%$ e valor preditivo negativo $=83 \%)$.

\section{ConClusão}

Considerando a boa concordância encontrada entre o diagnóstico da DHGNA realizado pela USG e a biópsia hepática, reforça a importância da condução de mais estudos com a utilização deste exame associado a parâmetros bioquímicos como forma de potencializar seu efeito diagnóstico e assim minimizar a necessidade da realização da biópsia hepática para diagnóstico de formas mais graves da doença.

\section{Agradecimento}

Este estudo foi apoiado pela FAPERJ e CNPq.

\section{Conflito de interesse: Não há}

\section{SUMMARY}

Abdominal Ultrasound and magnetic resonance imaging: A COMPARATIVE STUDY ON THE NON-ALCOHOLIC FATTY LIVER DISEASE DIAGNOSIS IN MORBIDLY OBESE PATIENTS

OBJECTIVES. To evaluate the concordance between abdominal ultrasound and an MRI (Magnetic Resonance Imaging) in the diagnosis of non-alcoholic fatty liver disease (NAFLD), and concordance of these two methods with the histopathological exam.
METHODS. The population studied was comprised of 145 patients with morbid obesity $\left(B M I \geq 40 \mathrm{Kg} / \mathrm{m}^{2}\right)$, of both genders. NAFLD diagnosis was performed by MRI and Ultrasound. Liver biopsy was performed in a sub-sample $(n=40)$. To evaluate the concordance of these two methods, the kappa coefficient was used.

RESULTS. Concordance between both methods (MRI and Ultrasound) was poor and not significant (Kappa adjusted $=0.27 ; \mathrm{Cl}$ $95 \%=0.07-0.39$.) Nevertheless a slight concordance was found between diagnosis of NAFLD by ultrasound and the hepatic biopsy, with 83.,3\% of concordant results and Kappa adjusted $=0.67$. Results of an MRI and the histopathological exam were compared and results showed $53.6 \%$ of concordant results and kappa adjusted $=0.07$.

CONCLUSION. The concordance found in the diagnosis performed using the ultrasound method and the hepatic biopsy, shows a need to implement and perform more research on the use of ultrasound to validate and reconsider these methods. This would minimize the need to perform biopsies to detect and diagnose such disease. [Rev Assoc Med Bras 2009; 55(I): 45-9]

KEY WORDS: Abdominal ultrasound. Magnetic resonance imaging. Morbid obesity. Non-alcoholic fatty liver disease.

\section{REFERÊNCIAS}

I. Harrison AS, Bisceglie AM. Advances in the understanding and treatment on nonalcoholic fatty liver disease. Drugs. 2003;63:2379-94.

2. Saadeh S, Younossi ZM, Remer EM, Gramlich T, Ong JP, Hurley M, et al. The utility of radiological imaging in nonalcoholic fatty liver disease. Gastroenterology. 2002; | 23:745-50.

3. Joy D, Thava VR, Scott BB. Diagnosis of fatty liver disease: is biopsy necessary? Eur J Gastroenterol Hepatol. 2003; I 5:539-43.

4. Ramesh S, Sanyal A. Evaluation and management of non-alcoholic steatohepatitis. J Hepatol. 2005;42:82-93.

5. Adams LA, Angulo P. Recent concepts in non-alcoholic fatty liver disease. Diabet Med. 2005;22:1 129-33.

6. Shimada M, Hashimoto E, Taniai M, Hasehawa K, Okuda H, Hayashi N, et al. Hepatocellular carcinoma in patients with non-alcoholic steatohepatitis. J Hepatol. 2002;37: I 54-60.

7. Sheth SC, Gordon FD, Chopra S. Nonalcoholic steatohepatitis. Ann Intern Med. 1997; 126:137-45.

8. Younossi Z, Diehl AM, Ong JP. Nonalcoholic fatty liver disease: an agenda for clinical research. Hepatology. 2002;35:746-52.

9. Cuppari L. Guias de medicina ambulatorial e hospitalar. Nutrição Clínica no adulto. UNIFESP. São Paulo: Manole; 2002.

10. World Health Organization. Obesity: preventing and managing the global epidemic. Report of a WHO Consultation on Obesity. WHO: Geneva; 1998.

I I. Pratt DS, Kaplan MM. Avaliação da função hepática. In: Braunwald E, Fauci AS, Kasper DL, Hauser SL, Longo DL, Jameson LR, editors. Harrison medicina interna. 15a ed. Rio de Janeiro: Editora Mc Graw Hill; 2002. p. $1813-6$

12. Brunt EM, Janney CG, Di Bisceglie AM, Neuschwander-Tetri BA, Bacon BR. Nonalcoholic steatohepatitis: a proposal for grading and staging the histological lesions. Am J Gastroenterol. 1999;94:2467-74.

13. Youssef WI, McCullough AJ. Steatohepatitis in obese individuals. Best Pract Res Clin Gastroenterol. 2002; 16:733-47.

14. Chitturi S, George J. Interaction of iron, insulin resistance, and nonalcoholic steatohepatitis. Curr Gastroenterol Rep. 2003;5: I 8-25.

15. Almeida AG. Correlações entre achados laboratoriais e doença hepática gordurosa não alcoólica em pacientes portadores de obesidade mórbida [dissertação] São Paulo: Universidade de São Paulo; 2005. 
16. Festi D, Colecchia A, Sacco T, Bondi M, Roda E, Marchesini G. Hepatic steatosis in obese patients: clinical aspects and prognostic significance. Obes Rev. 2004;5:27-42.

17. Brunt EM. Nonalcoholic steatohepatitis. Semin Liver Dis. 2004;24:3-20.

18. Angulo P. Nonalcoholic fatty liver disease. N Engl J Med. 2002; | 8: | 22 | -3 | .

19. Allard JP. Other disease associations with non-alcoholic fatty liver disease (NAFLD). Best Pract Res Clin Gastroenterol. 2002; I 6:783-95.

20. World Health Organization. Indicators for assessing vitamin A deficiency and their application in monitoring and avaluating intervention programmes. Micronutrient Series, WHO/NUT. I0. Geneva: World Health Organization; 1996.

21. Mottin CC, Moretto M, Padoin AV, Swarowsky AM, Toneto MG, Glock $\mathrm{L}$, et al. The role of ultrasound in the diagnosis of hepatic steatosis in morbidly obese patients. Obes Surg. 2004; |4:635-7.

22. Fris RJ. Preoperative low energy diet diminishes liver size. Obes Surg. 2004; | 4: | | 65-70.
23. Lewis MC, Phillips ML, Slavotinek JP, Kow L, Thompson CH, Toouli, J. Change in liver size and fat content after treatment with Optifast very low calorie diet. Obes Surg. 2006; 16:697-701.

24. Liang RJ, Wang HH, Lee WJ, Liew PL, Lin JT, Wu MS. Diagnostic value of ultrasonographic examination for nonalcoholic steatohepatitis in morbidly obese patients undergoing laparoscopic bariatric surgery. Obes Surg. 2007; 17:45-56.

25. Bernal-Reyes R, Bernal-Serrano D. Liver functional test (LFT) and ultrasound (US) usefulness in the diagnostic of non-alcoholic steatohepatitis (NASH). Rev Gastroenterol Mex. 2006;71:6-10.

Artigo recebido: 09/09/07

Aceito para publicação: 15/04/08 